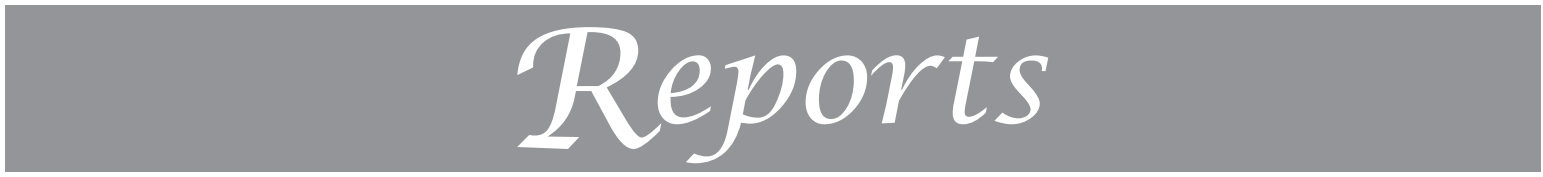

Ecology, 100(6), 2019, e02708

C) 2019 by the Ecological Society of America

\title{
Coexistence and competitive exclusion in mutualism
}

\author{
Christopher A. Johnson ${ }^{1,2,3}$ And Judith L. Bronstein ${ }^{1}$ \\ ${ }^{1}$ Department of Ecology and Evolutionary Biology, University of Arizona, P.O. Box 210088, Tucson, Arizona 85721 USA \\ ${ }^{2}$ Center for Adaptation to a Changing Environment, Institute of Integrative Biology, ETH Zürich, Universitätstrasse 16, \\ Zürich 8092 Switzerland
}

Citation: Johnson, C. A., and J. L. Bronstein. 2019. Coexistence and competitive exclusion in mutualism. Ecology 100(6):e02708. 10.1002/ecy.2708

\begin{abstract}
The competitive exclusion principle is fundamental to understanding coexistence. Well-established theories predict the conditions for coexistence in consumer-resource interactions. Given that species often compete for commodities offered by mutualists, competitive exclusion theory should also be critical to understanding how mutualisms function. We explicitly apply the competitive exclusion principle to mutualism and derive a rule analogous to Tilman's $R^{*}$ rule for exploitative competition. Coexistence is impossible when competitors compete solely for a shared partner-provided commodity because superior competitors deplete that commodity sufficiently to exclude inferior competitors. We then investigate how competition between two guild members for a partner-provided commodity and a resource external to the mutualism affects competitor coexistence. There are three key results. First, coexistence is possible via partitioning of a partner-provided commodity and another resource. Second, unlike in classic $R^{*}$ Theory, competitive outcomes are influenced both by species' abilities to obtain commodities and their mutualisms with the shared commodity-providing partner, which can indirectly alleviate competitors' commodity limitation. Third, the outcome of competition has important consequences for the commodity-providing partner, which depend on the type of mutualism and the competitive abilities of competing mutualists. This theory provides a novel framework for investigating how competitors for mutualistic commodities coexist in nature.
\end{abstract}

Key words: coexistence; competition; competitive exclusion principle; mutualisms; persistence; $\mathrm{R} *$ theory.

\section{INTRODUCTION}

A core concept in ecology is the competitive exclusion principle: complete competitors cannot coexist (Hardin 1960). Competitive exclusion can occur in consumerresource interactions as a result of either exploitative competition for limiting resources or apparent competition mediated through shared natural enemies. From a theoretical perspective, the conditions under which competitive exclusion occurs due to exploitative competition can be quantified by $R^{*}$ Theory, which predicts that multiple species cannot coexist on shared resources without niche differentiation (Tilman 1977, 1980, 1982). Species that persist at the lowest resource density $\left(R^{*}\right)$ competitively exclude all species that are most limited by

Manuscript received 28 July 2018; revised 29 January 2019; accepted 11 March 2019. Corresponding Editor: Sebastian J. Schreiber.

${ }^{3}$ E-mail: cjohnson@env.ethz.ch that resource. For apparent competition, $P^{*}$ Theory predicts that species that best tolerate antagonism by shared enemies and thus persist at the highest consumer density $\left(P^{*}\right)$ competitively exclude all species that are most sensitive to attack by that consumer (Holt 1977, Holt et al. 1994).

Competition also plays a central role in the ecology and evolution of mutualism (Jones et al. 2012). It is a key feature of generalized mutualisms involving guilds of species, which are far more common in nature than are one-to-one relationships (Stanton 2003). Mutualistic commodities are resources or services that increase the fitness of recipient species. In nature, the availability of commodities is generally limited, as producers usually incur some cost in providing them (e.g., Pringle 2016). Therefore, there is competition within and among species that share the same mutualistic partner for the commodities provided by that shared partner. For example, in pollination mutualisms, foraging animals that vector pollen compete both intraspecifically and, except in 
tightly specialized interactions, interspecifically for commodities such as nectar provided by a given plant species. Plants in turn compete intraspecifically and, again except in tightly specialized interactions, interspecifically for the attention of pollen-vectoring foragers. The positive net effects of mutualism are therefore mediated by competition on both sides of the interaction. In pollination mutualisms, for example, forager fitness resulting from nectar consumption and plant fitness resulting from pollen transfer depend critically on species' abilities to successfully forage and to successfully attract foragers, respectively. Competition for partnerprovided commodities occurs in tandem with competition for other limiting resources external to mutualism. For example, ant-defended plants compete not only for mutualistic protectors, but also for water and nutrients, and ants may compete for nesting sites and other prey (Lee and Inouye 2010, Palmer et al. 2013). Importantly, the interplay of competition and mutualism determines whether coexistence or exclusion arises.

From a theoretical perspective, few models explicitly consider competition for mutualistic commodities, and those that do have generated diverse and sometimes contrasting predictions. For example, some models predict that mutualistic interactions may undermine competitor coexistence by amplifying existing competitive asymmetries (e.g., Benadi 2015). Other studies predict that competitors engaging with shared mutualists may coexist when mutualists adaptively forage (e.g., Song and Feldman 2014, Revilla and Krrivan 2016), are sufficiently specialized (e.g., Benadi et al. 2013, Benadi 2015), or influence partners in a way that benefits competitors (e.g., Bever 2003, Lee and Inouye 2010). It is difficult, however, to distill concrete coexistence criteria from these models as do $R^{*}$ and $P^{*}$ theories for predator-prey interactions.

There is a conceptual gap between theory, which has generated relatively few predictions about the role of competition for mutualistic commodities in promoting or limiting coexistence, and empirical studies, which provide abundant evidence of competition for commodities in nature (reviewed by Palmer et al. 2003, Mitchell et al. 2009, Morales and Traveset 2009). Competition for commodities has been abundantly documented in mutualisms involving protection (e.g., Mooney and Mandal 2010), pollination (e.g., Mitchell et al. 2009), and seed dispersal (e.g., Alcántara et al. 1997). Competition for and among nutritional symbionts is also well-documented (Kiers et al. 2003, Bennett and Bever 2009). Indeed, competition for mutualists has clearly driven evolution of the commodities that attract and reward them (Jones et al. 2012).

Bridging this conceptual gap between theory and data requires frameworks that explicitly incorporate competition for mutualistic commodities, yield concrete criteria for coexistence and exclusion, and disentangle the effects of competition for commodities from those of competition for other resources. Such frameworks should address three questions. (1) How does competition within mutualistic guilds for shared partner-provided commodities affect the coexistence of members of that guild? The goal here is to derive explicit conditions for competitive exclusion in mutualistic guilds akin to $R^{*}$ and $P^{*}$ theories for consumer-resource interactions. (2) How does competition for mutualistic commodities interact with competition for other limiting resources to determine coexistence? The goal here is to understand whether competition for mutualistic commodities is fundamentally different from competition for other resources. If it is, how should theory incorporate competition for these commodities? (3) How does the outcome of competition affect commodity-providing partners? The goal here is to predict under which competitive regimes, coexistence or exclusion, shared partners attain the greatest benefits from competing mutualists. We derive models to investigate these three fundamental issues by applying the ideas underlying the competitive exclusion principle to mutualism.

\section{CONCEPTUAL Framework}

$R^{*}$ Theory predicts that competitive exclusion is the only outcome of exploitative competition between species sharing a single resource without niche differentiation (Tilman 1977, 1980, 1982). To explore when and how competing mutualists are able to coexist, we apply this prediction to mutualism by considering competition for a single mutualistic commodity. This provides a theoretical null expectation against which to evaluate more complex models. We then investigate how competition for both a mutualistic commodity and a different limiting resource affects coexistence of competing mutualists.

$R^{*}$ Theory considers both essential and substitutable resources (Tilman 1980, 1982). For essential resources, competitors require all resources and their population growth rate is determined by the most limiting resource. For substitutable resources, competitors can utilize resources interchangeably, and their population growth rate is determined by some contribution (usually additive) of all consumed resources. There are several key differences between mutualistic commodities and other resources (note that while commodities can be resources, to succinctly distinguish between the two, we hereafter refer to rewards or services obtained from mutualistic partners as "commodities" and all other resources as "resources"). Commodities and resources are not substitutable for many mutualisms. For example, some plant species can persist without the services of pollinator mutualists, but no plant species can persist only on those services. Conversely, a commodity and resource can both be essential for persistence, but population growth can be determined by some contribution of each, rather than by the most limiting quantity. We avoid the terms "essential" and "substitutable" but, following $R^{*}$ Theory, investigate cases in which population growth is determined by the most limiting commodity or resource (representing obligate mutualisms) and by the contributions of each (representing either obligate or facultative mutualisms depending on whether species persist without the commodity). 
A final key point is that not all commodities are amenable to being modeled as depletable quantities. We consider two broad categories of mutualisms: resourceexchange mutualisms, exemplified here by plant-mycorrhizae mutualisms (Fig. 1a), and resource-for-service mutualisms, exemplified here by plant-pollinator mutualisms (Fig. 1b). Resource-exchange mutualisms involve reciprocal exchange of nutritional commodities: for example, plants acquire mineral nutrients from mycorrhizae and mycorrhizae acquire carbohydrates from plants. The distinctive feature of this framework is that it explicitly models dynamics of commodities, such that species deplete commodities and are competitively excluded if commodities are sufficiently depleted. Resource-for-service mutualisms, in contrast, involve the exchange of nutritional commodities for services: for example, flower-foraging animals deplete commodities (usually nectar) provided by plants, and plants obtain pollination services from foragers. Services are more abstract quantities than are rewards, but can nonetheless constitute limiting factors for mutualists; pollinator limitation for plants is a classic example (Knight et al. 2005). Unlike mutualistic commodities, mutualistic services cannot accumulate; they must either be conferred or lost (mathematically, there is no equation for service commodities). Rather than depleting services, species compete for services by differentially attracting shared partners and thus depriving competitors of access to those services. Differences in the biology of resourceexchange and resource-for-service mutualisms necessitate distinct mathematical models. Importantly, the key results that we present here are largely robust, and often identical, between resource-exchange and resource-forservice models, suggesting that competition functions similarly in each despite differences in their underlying biology. We therefore present only resource-exchange models here. Resource-for-service models are presented in Appendix S1.

\section{a) Resource-exchange mutualisms}

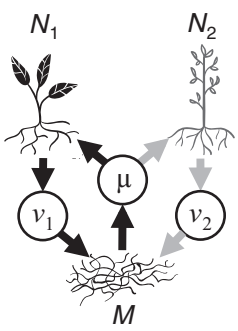

FIG. 1. Competition for commodities provided by a shared mutualistic partner in (a) resource-exchange mutualisms such as mycorrhizae and (b) resource-for-service mutualisms such as pollination. (a) Resource-exchange mutualisms: partner $M$ produces commodity $\mu$, which competitors $N_{i}$ deplete and provide commodities $v_{i}$ in return. (b) Resource-for-service mutualisms: competitors $N_{i}$ obtain services (solid arrows) by attracting and rewarding (dashed arrows) partner $M$.

\section{Model 1: Competition for Mutualistic Commodities} Alone

Consider two species $N_{i}$ that compete solely for a commodity $\mu$ from a shared mutualistic partner $M$ and that provide commodities $v_{i}$ in return (Fig. 1a), the dynamics of which are given by

$$
\begin{gathered}
\frac{1}{N_{i}} \frac{d N_{i}}{\mathrm{dt}}=e_{i} a_{i} \mu-d_{i} \\
\frac{1}{\mathrm{M}} \frac{d \mathrm{M}}{\mathrm{dt}}=\sum_{i} b_{i} a_{M i} v_{i}-q M+r_{M} \\
\frac{d \mu}{\mathrm{dt}}=p_{M} M-\delta_{\mu} \mu-\sum_{i} a_{i} \mu N_{i} \\
\frac{d v_{i}}{d t}=p_{i} N_{i}-\delta_{i} v_{i}-a_{M i} v_{i} \mathrm{M}
\end{gathered}
$$

where $a_{i}, e_{i}$, and $d_{i}$ are the commodity acquisition rate, conversion efficiency, and mortality rate of $N_{i}$, respectively; $a_{M i}$ and $b_{i}$ are the partner's acquisition rate and benefit of commodity $v_{i}$, respectively; $r_{M}$ and $q$ give partner intrinsic growth rate and density dependence, respectively; $p_{i}$ and $p_{M}$ are commodity production rates by $N_{i}$ and $M$, respectively; and $\delta_{i}$ and $\delta_{\mu}$ are commodity decay rates of $v_{i}$ and $\mu$, respectively (See Table 1 for parameter values). We assume that costs of producing commodities contribute to species' mortality rates such that costs factor into species' competitive abilities via $d_{i}$.

Assessing coexistence analytically for this model is challenging for many reasons. Evaluating mutual invasibility (whether species can increase in density when rare) is problematic because mutualisms are characterized by Allee effects that cause species to go extinct when rare. Furthermore, the models are sufficiently complex to render full stability analyses analytically intractable. We therefore determine the conditions for coexistence equilibria to exist and define Lyapunov functions that give necessary and sufficient conditions for their stability.

\section{How Competition for a Shared Commodity Affects Coexistence Within a Mutualistic Guild}

When species compete exclusively for a commodity provided by a shared mutualistic partner, coexistence requires that

$$
\frac{d_{1}}{e_{1} a_{1}}=\frac{d_{2}}{e_{2} a_{2}}
$$

Thus, coexistence is impossible except in the exceedingly rare case in which both competitors' commodity acquisition rates, conversion efficiencies, and mortalities are all perfectly balanced. Defining the equilibrium commodity $\mu^{*}\left(=d_{i} / e_{i} a_{i}\right)$, the key prediction here is that the 
TABLE 1. Model variables and parameters with descriptions and values are given for resource-exchange (Eqs. 1, 3, and 4) and resource-for-service (Appendix S1: Eqs. S1 and S2) mutualisms.

\begin{tabular}{|c|c|c|c|c|}
\hline Symbol & Description & $\begin{array}{l}\text { Value (Eq. } 2) \\
\text { commodity only }\end{array}$ & $\begin{array}{l}\text { Value (Eq. 4a) } \\
\text { "essential" }\end{array}$ & $\begin{array}{l}\text { Value (Eq. 4b) } \\
\text { "substitutable" }\end{array}$ \\
\hline$N_{i}$ & competitor density & - & - & - \\
\hline$M$ & partner density & - & - & - \\
\hline$\mu \dagger$ & commodity of $M$ & - & - & - \\
\hline$v_{i} \dagger$ & commodity of $N_{i}$ & - & - & - \\
\hline$R$ & resource availability & - & - & - \\
\hline$e_{i}$ & commodity conversion efficiency & $e_{1}=0.5, e_{2}=1$ & $e_{1}=0.5, e_{2}=1$ & $e_{1}=0.5, e_{2}=1$ \\
\hline$\varepsilon_{i}$ & resource conversion efficiency & - & $\varepsilon_{1}=1, \varepsilon_{2}=0.5$ & $\varepsilon_{1}=1, \varepsilon_{2}=0.5$ \\
\hline$a_{i}$ & $\mu$ acquisition rate & varied & $a_{2}=0.1 \S$ & $a_{2}=0.09 \S$ \\
\hline$\alpha_{i}$ & $R$ acquisition rate & - & $\alpha_{1}=0.1$ ฯ & $\alpha_{1}=0.09 q$ \\
\hline$d_{i}$ & per capita mortality rate of $N_{i}$ & 0.1 & $0.01 \dagger, \#, 0.1 \dagger, \|$ or $0.01 \ddagger$ & 0.1 \\
\hline$r_{M}$ & growth rate of $M$ & 0.1 & 0.1 & 0.1 \\
\hline$q$ & self-limitation in species $M$ & 1 & $0.001 \dagger, \#, 0.004 \dagger, \|$ or $1 \dagger$ & $1 \dagger$ or $0.1 \ddagger$ \\
\hline$b_{i}$ & mutualist quality of $N_{i}$ & 1 & 1 & 1 \\
\hline$a_{M i} \dagger$ & $v_{i}$ acquisition rate & 1 & 1 & 1 \\
\hline$p_{i} \dagger$ & commodity $v_{i}$ production rate & 1 & $p_{1}=0.15 \#, p_{2}=0.1 \# ; p_{1}=1.5\left\|, p_{2}=1\right\|$ & 1 \\
\hline$\delta_{i} \dagger$ & commodity decay rate & 1 & 1 & 1 \\
\hline$p_{M} \dagger$ & $\mu$ production rate & 1 & 0.006 & 1 \\
\hline$\delta_{\mu} \dagger$ & $\mu$ decay rate & 1 & 1 & 1 \\
\hline$\tau_{i} \ddagger$ & interaction time & 1 & 1 & 1 \\
\hline$r_{R}$ & $R$ supply rate & - & 1 & 1 \\
\hline$S_{R}$ & $R$ supply constant & - & 1 & 1 \\
\hline$s_{R i}$ & $R$ stoichiometric coefficient & - & 1 & - \\
\hline$s_{\mu i}$ & $\mu$ stoichiometric coefficient & - & 1 & - \\
\hline
\end{tabular}

$\dagger$ Resource-exchange mutualisms.

Resource-for-service mutualisms

$\S$ Inferior competitor for commodity ( $a_{2}$ varied in figures).

Inferior competitor for resource ( $\alpha_{1}$ varied in figures).

\# Shared partner is facultative.

\| Shared partner is obligate.

- The variable or parameter does not have a fixed value (i.e., either it is not in the model or it is a state variable).

mutualist that can best acquire the shared commodity (lowest $\mu^{*}$ ) excludes all inferior competitors that are most limited by that commodity (see Appendix S2 for mathematical details). Appendix S3 shows that the same conditions arise within resource-for-service mutualisms: the mutualist that can best attract the shared, serviceproviding partner excludes all competitors that are most limited by that service. Exclusion occurs because the competitively dominant mutualist limits the commodity sufficiently that the inferior competitor cannot maintain a positive per capita growth rate. These results constitute the first formal application of $R^{*}$ Theory to the case of mutualism.

\section{Model 2: Competition for Both a Mutualistic Commodity And a Limiting Resource}

Species in nature compete for resources other than just mutualist-provided commodities. Classic theory shows that competing consumers can coexist on multiple resources (e.g., Tilman 1977, Chesson 2000). Can competing mutualists coexist when limited by a shared commodity and a single resource? Consider when species compete for a commodity and a limiting resource $R$ that is external to the mutualism; e.g., water. The dynamics of competitors $N_{i}$ and a resource $R$ and commodity $\mu$ for which they compete are given by

$$
\begin{gathered}
\frac{1}{N_{i}} \frac{d N_{i}}{\mathrm{dt}}=G_{i}-d_{i} \\
\frac{d \mathrm{R}}{\mathrm{dt}}=r_{R}\left(S_{R}-\mathrm{R}\right)-\sum_{i} s_{\mathrm{R} i} F_{\mathrm{R} i} \mathrm{~N}_{i} \\
\frac{d \mu}{\mathrm{dt}}=p_{M} \mathrm{M}-\delta_{\mu} \mu-\sum_{i} s_{\mu i} F_{\mu i} N_{i}
\end{gathered}
$$

where $r_{R}$ and $S_{R}$ are the supply rate and constant of the resource, which is assumed to be abiotic. The functions $F_{R i}$ and $F_{\mu i}$ describe resource and commodity acquisition, respectively, where $s_{R i}$ and $s_{\mu i}$ are stoichiometric coefficients and the function $G_{i}$ translates acquisition to population growth. We investigate two cases. The first is when population growth is determined by the most limiting resource or commodity; for example, plant 
growth is constrained by either mineral nutrients from mycorrhizae or water, depending on which is most limiting. The second case is when the commodity and resource contribute additively to population growth; for example, plants are limited by both water and mineral nutrients from mycorrhizae, but mycorrhizae may or may not be required. Mathematically, these cases are given by

$$
\begin{gathered}
G_{i}=F_{R i}=F_{\mu i}=\min \left[\varepsilon_{i} \alpha_{i} \mathrm{R}, e_{i} a_{i} \mu\right] \\
\text { and } G_{i}=\varepsilon_{i} \alpha_{i} \mathrm{R}+e_{i} a_{i} \mu, F_{R i}=\alpha_{i} \mathrm{R}, F_{\mu i}=a_{i} \mu
\end{gathered}
$$

where $\alpha_{i}$ and $\varepsilon_{i}$ are, respectively, the acquisition rate and conversion efficiency of the resource. The dynamics of partner $M$ and commodities $v_{i}$ are given by Eqs. $1 \mathrm{~b}$ and $1 \mathrm{~d}$, respectively.

\section{How Competition for a Mutualistic Commodity and Another Resource Affects Coexistence}

The models are sufficiently complex to render full stability analyses intractable; however, there are three necessary (but not sufficient) conditions for coexistence (Tilman 1980). Each species must (1) be most limited by a different factor and (2) obtain proportionally more of this limiting factor. Last, (3) the supply of the commodity and resource must not disproportionately favor one competitor over the other. Species are most limited by different factors (condition 1) if

$$
\frac{d_{i}}{e_{i} a_{i}}>\frac{d_{j}}{e_{j} a_{j}} \text { and } \frac{d_{j}}{\varepsilon_{j} \alpha_{j}}>\frac{d_{i}}{\varepsilon_{i} \alpha_{i}}
$$

where $N_{i}$ is more commodity limited (greater $\mu^{*}$ or $M^{*}$ ) and $N_{j}$ is more resource limited (greater $R^{*}$ ). For commodity and resource supply to not disproportionately favor a species (condition 3 ):

$$
\frac{a_{i}}{\alpha_{i}}>\frac{p_{M} \mathrm{M}^{*}}{\delta_{\mu} S_{R}}>\frac{a_{j}}{\alpha_{j}}
$$

where the outer inequalities $\left(\frac{a_{i}}{\alpha_{i}} \frac{a_{j}}{\alpha_{j}}\right)$ insure that each species obtains proportionally more of the commodity or resource that most limits its per capita growth rate (condition 2). (In resource-for-service mutualisms, $\frac{p_{M} M^{*}}{\delta_{M} S_{R}}$ becomes $\frac{M^{*}}{S_{R}}$ ). Parameter space plots verify that coexistence is possible only within the space delineated by Eq. 5, both when population growth is set by the most limiting commodity or resource and when the commodity and resource contribute additively to population growth (Fig. 2; see Appendices S4-S7: Fig. S1). The key points are that commodities can constitute limiting factors and coexistence requires partitioning of the commodity and resource.

Importantly, Eqs. 5 and 6 are necessary, but not sufficient, conditions for coexistence. As full stability criteria are analytically intractable, we systematically vary two key parameters related to the mutualisms: partner self limitation, $q$, and community production rate, $p_{M}$, in resource-exchange mutualisms or per capita growth rate, a) Most limiting commodity or resource

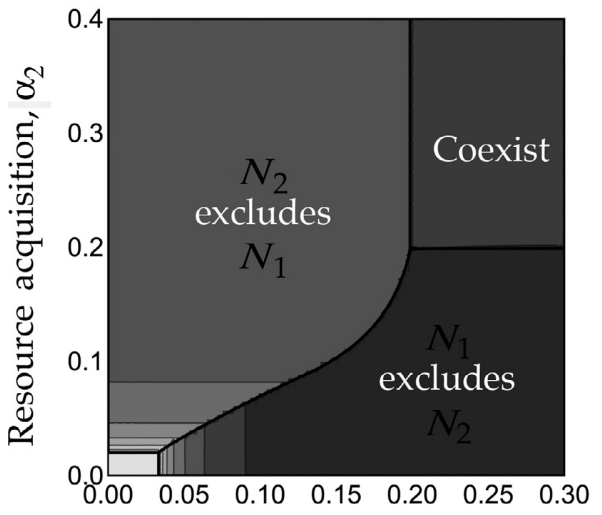

b) Additive commodity and resources

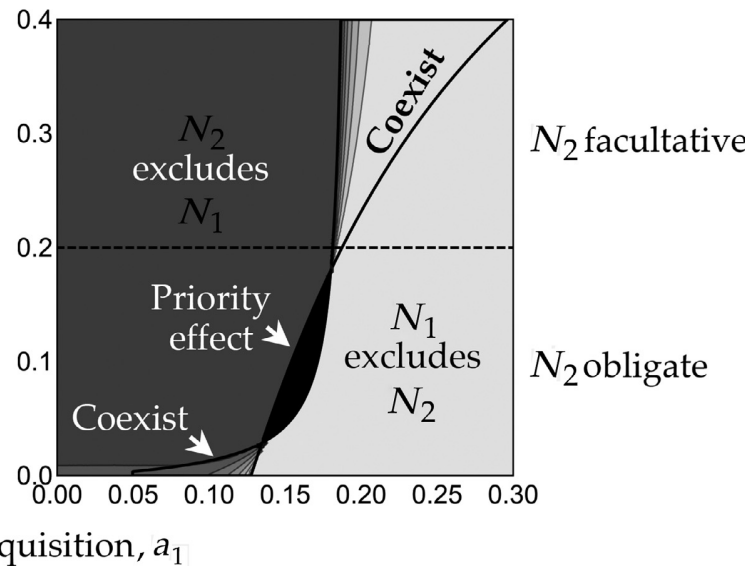

FIG. 2. Parameter space plots illustrate the outcome of competition in resource-exchange mutualisms when competitor population growth is determined by (a) the most limiting mutualistic commodity or limiting resource and determined by (b) the additive contributions of the commodity and resource and the shared mutualistic partner is facultative. Commodity acquisition rate of $N_{1}$ $\left(a_{1}\right)$ is plotted on the $x$-axis and resource acquisition rate of $N_{2}\left(\alpha_{2}\right)$ is plotted on the $y$-axis. In panel b, $N_{1}$ is obligate and $N_{2}$ is obligate below the dashed horizontal line and facultative above. Darker gray shades indicate greater partner density and thus more favorable outcomes of competition for the shared partner. Black lines delineate between competitive regimes: $N_{2}$ competitively excludes $N_{1} ; N_{1}$ competitively excludes $N_{2}$; and competitors coexist. A priority effect ensues in the black region in panel b in which competitive dominance and partner density depend upon initial competitor densities. Figures generated using Wolfram Mathematica (v. 11.3; Wolfram Research, Champaign, Illinois, USA). Parameters are given in Table 1. 
$r_{M}$, in resource-for-service mutualisms. Coexistence further requires that the shared partner is sufficiently limited that it cannot produce enough commodities to favor the commodity-limited competitor (see Appendices S4-S7: Fig. S2).

There are important differences between mutualistic commodities and other limiting resources such that care must be taken when applying $R^{*}$ Theory to mutualisms. In classic consumer-resource interactions, competitors cannot affect resource supply; they only deplete the resource such that the species that persists at the lowest resource density $R^{*}\left(=d_{i} / \varepsilon_{i} \alpha_{i}\right)$ excludes all other competitors most limited by that resource. This is Tilman's $R^{*}$ rule (Tilman 1977, 1982). In mutualisms, however, species can influence the availability of the commodity for which they compete in two ways: by directly obtaining commodities, and by indirectly buoying commodity supply via their mutualisms with the shared partner. By conferring mutualistic benefits to the shared partner, competitors buoy partner density, which in turn leads to greater commodity production, effectively alleviating competitors' commodity limitation.

\section{How the Outcome of Competition Affects a Commodi- ties-Providing Mutualistic Partner}

Since competitors for commodities are mutualists of their shared partner, the outcome of competition has important consequences for that shared partner. Not surprisingly, the outcome of competition is more favorable for the shared partner when competitive ability $\left(a_{i}\right)$ and mutualist quality $\left(b_{i}\right)$ are positively associated than when the superior competitor is a low-quality partner. When competitors are similarly rewarding $\left(b_{1}=b_{2}\right)$, partner density is greatest when the more commoditylimited competitor is excluded $\left(N_{2}\right.$ excludes $\left.N_{1}\right)$ in resource-exchange mutualisms when population growth is set by the most limiting commodity or resource (Fig. 2a; Appendix S4: Fig. S1) and in resource-for service mutualisms (Appendices S5 and S7: Fig. S1). Here, the less commodity-limited species attains greater density and thus yields greater commodities (or more interactions in resource-for-service mutualisms) to the shared partner. Conversely, partner density is greatest when the less commodity-limited competitor is excluded $\left(N_{1}\right.$ excludes $N_{2}$ ) in resource-exchange mutualisms when the commodity and resource contribute additively to population growth (Fig. 2b; Appendix S6: Fig. S1). Here, the more commodity-limited competitor must provide commodities at greater rates $\left(p_{1}>p_{2}\right)$ to persist, thus buoying partner fitness. In sum, these results suggest that the outcome of competition has important consequences for partners of competing mutualists.

\section{DISCUSSION}

The idea of competitive exclusion is central to understanding species coexistence (Hardin 1960, Tilman 1977,
1980, 1982). Long-standing theories predict when competitive exclusion occurs in exploitative (Tilman 1977, 1980, 1982) and apparent competition (Holt 1977, Holt et al. 1994). While a role for competitive exclusion theory is well established within consumer-resource interactions, it has yet to be explicitly considered in mutualism theory. This conceptual gap is surprising given that competition appears to be central to the ecological and evolutionary dynamics of mutualism (Jones et al. 2012).

Here, we formally apply the ideas underlying the competitive exclusion principle to mutualism. We investigated three overarching questions. First, how does competition within mutualistic guilds for partner-provided commodities affect coexistence of members of that guild? Theory presented here extends the competitive exclusion principle to mutualism: complete competitors for mutualistic commodities cannot coexist. The species that persists on the lowest commodity availability excludes all other competitors (Eq. 2). This result is Tilman's $R^{*}$ Theory applied to mutualism. Second, how does competition for both mutualistic commodities and other limiting resources affect coexistence? Coexistence is possible when competitors partition a commodity and a resource (Eqs. 5 and 6). Unlike in classic $R^{*}$ Theory, competitors indirectly alter commodity availability via their mutualisms with the commodity-providing partner. The key point is that while the competitive exclusion principle operates in mutualism, one cannot simply apply $R^{*}$ Theory to mutualism without considering how the mutualisms between competitors and a shared mutualist partner feed back to alter competition. Finally, we asked how the outcome of competition affects commodity-providing partners. Our theory predicts that the outcome of competition can have important consequences for partners of competing mutualists. The outcome of competition is generally more favorable to a shared partner when a less commodity-limited species, which attains greater density, is competitively dominant. Only when the commodity and resource contribute additively to population growth in resource-exchange mutualisms is it more favorable to a shared partner when a more commodity-limited species is competitively dominant.

From an empirical perspective, surprisingly little is known with regard to whether the best competitors for mutualistic commodities are also the best mutualists, pointing to a critical gap in the literature. Mooney and Mandal (2010) showed in an ant-aphid protection mutualism that the competitively dominant ant species also provided 13-fold greater benefits to their aphid mutualists. This is perhaps not surprising in a protection mutualism, as ant aggression is likely key to both competitive dominance and to effectiveness at defending partners. How does competitive ability relate to mutualistic quality in interactions where aggression is not key to mutualistic service, such as in transportation and nutrition mutualisms? Indeed, Bennett and Bever (2009) demonstrated that the most beneficial mycorrhizal fungus species for Plantago lanceolata is the worst competitor 
for root space, whereas the worst fungal mutualist is the best competitor for $P$. lanceolata roots. Theory predicts that mutualistic soil microbes can promote the coexistence of competing plant species when microbes exert less positive effects on their associated plant species than on a competing plant species (Bever et al. 1997, Bever 2003). In this framework, there are multiple mutualistic partners (soil microbe communities) and plants do not directly compete for commodities provided by soil microbes as in our theory. Nevertheless, both theories provide complementary predictions that coexistence requires that interactions with a shared mutualistic partner do not disproportionately favor one competitor over another.

Given that the availability of mutualistic commodities is generally limited in nature, we expect competition within and among species for partner-provided commodities to be important for the persistence of most mutualisms in nature. Empirical data suggest that competition is in fact ubiquitous within mutualism (Palmer et al. 2003, Mitchell et al. 2009, Morales and Traveset 2009). Indeed, there is growing evidence (and concerns) that invasive mutualists may competitively displace native mutualists (e.g., Grove et al. 2017, but see Charlebois and Sargent 2017). Yet, while it is widely recognized that species compete for commodities, the consistency and strength of competition are rarely measured (Jones et al. 2012). A critical empirical hurdle is how to quantify competition for commodities in nature. Theory we present here provides a relatively simple metric (Eq. 2): superior competitors are mutualists that better acquire commodities (greater $a_{i}$ ) or more efficiently utilize commodities (greater $e_{i}$ ) relative to mortality rate, $d_{i}$. Quantifying these factors in nature should allow the strength of competition for commodities to be evaluated empirically. We predict that species with a strong potential to compete (co-flowering plants, for example) should be most limited by, and strong competitors for, different mutualistic commodities or other limiting resources. Studies that explicitly investigate competition for commodities in nature are needed to better understand the role of competition in mutualism and evaluate the threats posed to native mutualists by invasive competitors for mutualistic commodities.

Perhaps the most significant effect that the competitive exclusion principle has had on the field of ecology is that it has stimulated decades of seminal research on mechanisms promoting coexistence in the face of competitive exclusion (Chesson 2000). In contrast, mutualism theory has generated relatively few advances in understanding how competitors for commodities coexist. Indeed, the vast majority of mutualism models do not mechanistically consider competition for mutualistic commodities (Johnson and Amarasekare 2013), either because they focus on tightly coupled pairwise mutualisms in which interspecific competition is irrelevant, or because commodities are implicitly modeled such that they do not constitute limiting factors. There has been recent theoretical interest in coexistence of mutualists that compete for shared commodities (Lee and Inouye 2010, Benadi et al. 2012, 2013, Song and Feldman 2014, Benadi 2015, Revilla and Krrivan 2016). These models, however, do not yield clear criteria for competitive exclusion, as $R^{*}$ and $P^{*}$ theories do for other consumerresource interactions. By explicitly considering competition for commodities, this theory yields a null expectation of competitive exclusion against which to test coexistence mechanisms. The next step is to consider multiple mutualistic partners and other limiting resources that characterize mutualisms in nature. The theory presented here provides a theoretical foundation upon which to develop new models for investigating mechanisms promoting coexistence of competitors for mutualistic commodities.

\section{ACKNOWLEDGMENTS}

We thank Sebastian Schreiber, Bob Holt, Christopher Klausmeier, Jonathan Levine, members of the Bronstein lab at the University of Arizona, and many anonymous reviewers for their helpful comments. We thank Julie Johnson for illustrations in Fig. 1. This research was supported by Science Foundation Arizona Grant No. BSP0528-13 and the ETH Zürich Center for Adaptation to a Changing Environment to C. Johnson. C. Johnson developed the models and C. Johnson and J. Bronstein wrote the manuscript.

\section{Literature Cited}

Alcántara, J. M., P. J. Rey, F. Valera, A. M. Sánchez-Lafuente, and J. E. Gutiérrez. 1997. Habitat alteration and plant intraspecific competition for seed dispersers. An example with Olea europaea var. sylvestris. Oikos 79:291-300.

Benadi, G. 2015. Requirements for plant coexistence through pollination niche partitioning. Trends in Ecology and Evolution 282:1-9.

Benadi, G., N. Blüthgen, T. Hovestadt, and H.-J. Poethke. 2012. Population dynamics of plant and pollinator communities: stability reconsidered. American Naturalist 179:157-168.

Benadi, G., N. Blüthgen, T. Hovestadt, and H.-J. Poethke. 2013. When can plant-pollinator interactions promote plant diversity? American Naturalist 182:131-146.

Bennett, A. E., and J. D. Bever. 2009. Trade-offs between arbuscular mycorrhizal fungal competitive ability and host growth promotion in Plantago lanceolata. Oecologia 160:807-816.

Bever, J. D. 2003. Soil community feedback and the coexistence of competitors: conceptual frameworks and empirical tests. New Phytologist 157:465-473.

Bever, J., K. Westover, and J. Antonovics. 1997. Incorporating the soil community into plant population dynamics: the utility of the feedback approach. Journal of Ecology 85:561-573.

Charlebois, J. A., and R. D. Sargent. 2017. No consistent pollinator-mediated impacts of alien plants on natives. Ecology Letters 20:1479-1490.

Chesson, P. 2000. Mechanisms of maintenance of species diversity. Annual Review of Ecology and Systematics 31:343-358.

Grove, S., K. A. Haubensak, C. Gehring, and I. M. Parker. 2017. Mycorrhizae, invasions, and the temporal dynamics of mutualism disruption. Journal of Ecology 105:1496-1508.

Hardin, G. 1960. The competitive exclusion principle. Science 131:1292-1297.

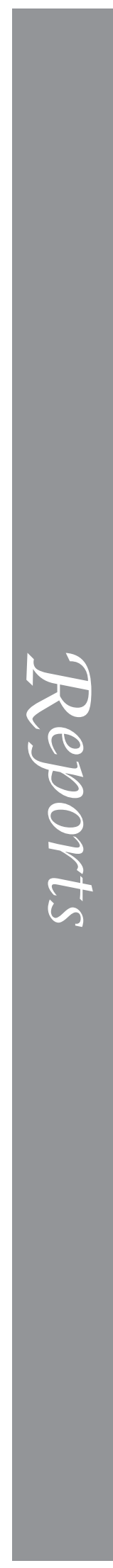


Holt, R. 1977. Predation, apparent competition, and the structure of prey communities. Theoretical Population Biology 12:197-229.

Holt, R., J. Grover, and D. Tilman. 1994. Simple rules for interspecific dominance in systems with exploitative and apparent competition. American Naturalist 144:741-771.

Johnson, C. A., and P. Amarasekare. 2013. Competition for benefits can promote the persistence of mutualistic interactions. Journal of Theoretical Biology 328:54-64.

Jones, E. I., J. L. Bronstein, and R. Ferrière. 2012. The fundamental role of competition in the ecology and evolution of mutualisms. Annals of the New York Academy of Sciences 1256:66-88.

Kiers, E., R. Rousseau, S. West, and R. Denison. 2003. Host sanctions and the legume-rhizobium mutualism. Nature 425:78-81.

Knight, T. M., J. A. Steets, J. C. Vamosi, S. J. Mazer, M. Burd, D. R. Campbell, M. R. Dudash, M. O. Johnston, R. J. Mitchell, and T.-L. Ashman. 2005. Pollen limitation of plant reproduction: pattern and process. Annual Review of Ecology, Evolution, and Systematics 36:467-497.

Lee, C. T., and B. D. Inouye. 2010. Mutualism between consumers and their shared resource can promote competitive coexistence. American Naturalist 175:277-288.

Mitchell, R. J., R. J. Flanagan, B. J. Brown, N. M. Waser, and J. D. Karron. 2009. New frontiers in competition for pollination. Annals of Botany 103:1403-1413.

Mooney, K. A., and K. Mandal. 2010. Competition hierarchies among ants and predation by birds jointly determine the strength of multi-species ant-aphid mutualisms. Oikos 119:874-882.
Morales, C. L., and A. Traveset. 2009. A meta-analysis of impacts of alien vs. native plants on pollinator visitation and reproductive success of co-flowering native plants. Ecology Letters 12:716-728.

Palmer, T. M., M. L. Stanton, and T. P. Young. 2003. Competition and coexistence: exploring mechanisms that restrict and maintain diversity within mutualist guilds. American Naturalist 162:63-79.

Palmer, T. M., M. L. Stanton, T. P. Young, J. S. Lemboi, J. R. Goheen, and R. M. Pringle. 2013. A role for indirect facilitation in maintaining diversity in a guild of African acacia ants. Ecology 94:1531-1539.

Pringle, E. G. 2016. Integrating plant carbon dynamics with mutualism ecology. New Phytologist 210:71-75.

Revilla, T. A., and V. Křivan. 2016. Pollinator foraging flexibility and coexistence of competing plants. PLoS ONE 440:1-29.

Song, Z., and M. W. Feldman. 2014. Adaptive foraging behaviour of individual pollinators and the coexistence of co-flowering plants. Proceedings of the Royal Society B 281:20132437.

Stanton, M. L. 2003. Interacting guilds: moving beyond the pairwise perspective on mutualisms. American Naturalist 162:10-23.

Tilman, D. 1977. Resource competition between plankton algae: an experimental and theoretical approach. Ecology 58:338-348.

Tilman, D. 1980. Resources: a graphical-mechanistic approach to competition and predation. American Naturalist 116:362-393.

Tilman, D. 1982. Resource competition and community structure. Princeton University Press, Princeton, New Jersey, USA.

\section{SUPPORTING INFORMATION}

Additional supporting information may be found in the online version of this article at http://onlinelibrary.wiley.com/doi/ 10.1002/ecy.2708/suppinfo 